\title{
Magnetic field dependence of microwave radiation in intermediate-length Josephson junctions
}

Sørensen, Mads Peter; Parmentier, R. D.; Christiansen, Peter Leth; Skovgaard, Ove; Dueholm, B.; Jørgensen, E.; Koshelets, V. P.; Levring, O. A.; Monaco, Roberto; Mygind, Jesper

Total number of authors:

12

Published in:

Physical Review B

Link to article, DOI:

10.1103/PhysRevB.30.2640

Publication date:

1984

Document Version

Publisher's PDF, also known as Version of record

Link back to DTU Orbit

Citation $(A P A)$ :

Sørensen, M. P., Parmentier, R. D., Christiansen, P. L., Skovgaard, O., Dueholm, B., Jørgensen, E., Koshelets, V. P., Levring, O. A., Monaco, R., Mygind, J., Pedersen, N. F., \& Samuelsen, M. R. (1984). Magnetic field dependence of microwave radiation in intermediate-length Josephson junctions. Physical Review B, 30(5), 26402648. https://doi.org/10.1103/PhysRevB.30.2640

\section{General rights}

Copyright and moral rights for the publications made accessible in the public portal are retained by the authors and/or other copyright owners and it is a condition of accessing publications that users recognise and abide by the legal requirements associated with these rights.

- Users may download and print one copy of any publication from the public portal for the purpose of private study or research.

- You may not further distribute the material or use it for any profit-making activity or commercial gain

- You may freely distribute the URL identifying the publication in the public portal 


\title{
Magnetic field dependence of microwave radiation in intermediate-length Josephson junctions
}

\author{
M. P. Soerensen, R. D. Parmentier, P. L. Christiansen, and O. Skovgaard \\ Laboratory of Applied Mathematical Physics, The Technical University of Denmark, DK-2800 Lyngby, Denmark
}

\author{
B. Dueholm, E. Joergensen, V. P. Koshelets, O. A. Levring, R. Monaco, J. Mygind, \\ N. F. Pedersen, and M. R. Samuelsen \\ Physics Laboratory I, The Technical University of Denmark, DK-2800 Lyngby, Denmark
}

(Received 21 December 1983)

\begin{abstract}
Experimental measurements of current-voltage structure and emitted $X$-band radiation in applied magnetic field from overlap-geometry Josephson tunnel junctions of normalized length about 2 are compared with numerical simulations obtained with the use of a perturbed sine-Gordon model. The simulations furnish the current and field dependence of the oscillation configuration, from which can be calculated average voltages, frequencies, and power spectra. Simulation and experimental results are in good agreement with regard to the lobe structure of the height of the first zero-field step and/or second Fiske step in magnetic field and the field dependence of the radiation frequency within the various lobes, including details such as hysteresis between lobes. The simulations predict an alternation of the dominant frequency component with increasing field that accounts well for the experimental observations. The usefulness and limitations of cavity-mode analyses, both singlemode and multimode, are evidenced by comparison with the simulation results.
\end{abstract}

\section{INTRODUCTION}

Fluxon (soliton) propagation ${ }^{1}$ has been by now established as the essential physical mechanism underlying many of the observed experimental properties of long Josephson tunnel junctions. In particular, the appearance of both zero-field steps (ZFS's) and Fiske steps (FS's) in the current-voltage $(I-V)$ characteristics of such junctions and the emission of microwave radiation from junctions when biased on these steps seem to be explainable in terms of fluxon dynamics. ${ }^{2-5}$ A number of different approaches have been employed in the literature to account for the available experimental observations. These include perturbative expansions of the basic soliton equation involved (sine-Gordon equation), ${ }^{6}$ analytic extensions of small-junction theory (cavity-mode-interaction analyses), ${ }^{7}$ and mechanical analog ${ }^{8}$ and digital computer ${ }^{9}$ simulations (the references cited are intended to be representative, not exhaustive). Moreover, direct dynamic measurements at the single-fluxon level are beginning to appear in the literature. ${ }^{10,11}$

The perturbative approach is most suited for studying the behavior of low-order steps on very long junctions, inasmuch as the usual point of departure here consists of the exact analytic solutions of the sine-Gordon equation on the infinite spatial interval. Multimode extensions of small-junction theory, on the other hand, should presumably be most appropriate for relatively short junctions. For junctions which are neither very long nor very short, one would expect a priori that neither of these two approaches could be counted on to give reliable results. In such cases, direct simulation would seem to be indispensable.

The present paper is an attempt to elucidate further and in more detail just this case, viz., the dynamics underlying the behavior of intermediate-length Josephson junctions. To this end we compare the results of new experimental measurements of $I-V$ structure and microwave emission in magnetic field with the results of detailed numerical simulations. The agreement that emerges is quite convincing. For simplicity, attention is focused primarily on the first zero-field step (ZFS1) and on the second Fiske step (FS2) in junctions of normalized length of about 2. Since the voltage positions of ZFS1 and FS2 approximately coincide, we refer in the following to the step ZFS1/FS2. The numerical simulations are compared also with approximate analytic results, and the usefulness and limitations of the latter are clarified.

The paper is structured as follows: Section II contains a description of the mathematical model used and the techniques employed in its analysis. The results of this analysis are presented in Sec. III. The experimental measurements performed are described and discussed in Sec. IV. Finally, Sec. V contains our concluding comments.

\section{MATHEMATICAL MODEL AND COMPUTATION TECHNIQUES}

The mathematical model studied is the perturbed sineGordon equation, ${ }^{9}$

$$
\begin{aligned}
& \phi_{x x}-\phi_{t t}-\sin \phi=\alpha \phi_{t}-\beta \phi_{x x t}-\gamma, \\
& \phi_{x}(0, t)=\phi_{x}(L, t)=\eta,
\end{aligned}
$$

appropriate to an overlap-geometry junction. ${ }^{12}$ Here, $\phi$ is the usual Josephson-phase variable, $x$ is distance along the junction normalized to the Josephson penetration depth $\lambda_{J}, t$ is time normalized to the inverse of the Josephson 
plasma (angular) frequency $\omega_{0}$, and subscripts denote partial derivatives (see Ref. 9 for details of the normalizations). The model contains five parameters: $\alpha, \beta, \gamma, L$, and $\eta$. The term in $\alpha$ represents shunt (quasiparticle) loss. The $\beta$ term models dissipation due to the surface resistance of the superconducting films. The constant $\gamma$ measures a uniformly distributed bias current normalized to the maximum zero-voltage Josephson current. The constant $\eta$ is a normalized measure of the external magnetic field which determines the boundary conditions [Eq. (1b)] at the two ends of the junction of normalized length $L$. In this numerical study the dissipative and length parameters were fixed at $\alpha=0.05, \beta=0.02$, and $L=2$. These were chosen to be representative of typical physical values without, however, modeling any one specific junction. The bias current and magnetic field parameters were varied in the ranges $0 \leqq \gamma \leqq 1,0 \leqq \eta \lesssim 6$.

Equations (1) were integrated from initial conditions either similar to Eqs. (2) of Ref. 13 or (more often) using the final $\phi$ and $\phi_{t}$ distributions from runs at nearby points in parameter space. The integration was carried out using the implicit finite-difference method described in detail in Ref. 9, with space and time intervals set to 0.02 and 0.01 , respectively. Numerical accuracy and stability were verified by halving and doubling these intervals. During each run the time-averaged value of the voltage at the two ends of the junction and the power spectrum of the voltage at the left $(x=0)$ end were calculated. Here, voltage is defined as $\phi_{t}$, which represents the physical voltage normalized to $\hbar \omega_{0} / 2 e$, where $\hbar$ is Planck's constant divided by $2 \pi$, and $e$ is the magnitude of the electronic charge. These quantities were calculated over an integral number of oscillation periods during the last approximately 50 normalized time units of each run. The power spectra were calculated by means of a fast Fourier transform using a simple rectangular window. ${ }^{14}$ The values of $\left\langle\phi_{t}\right\rangle$ were calculated both from the elementary definition of average and the zero-frequency components of the power spectra.

Two checks were employed to assure that the average voltages and the power spectra were calculated over steady-state, not transient, dynamic configurations: (i) The values of $\left\langle\phi_{t}\right\rangle$ at the two junction ends were compared. Physically and mathematically, the time-averaged voltage must be constant along the length of the junction. (ii) The quantity $\left\langle\phi_{t}\right\rangle / 2 \pi f$, where $f$ is the fundamental oscillation frequency, was calculated. From the Josephson frequency relation, this quantity, in steady state, must be an integer whose value ( 1 or 2 in the present case) depends upon the type of oscillation present. ${ }^{8}$ If either of these conditions was not satisfied to within specified limits the time duration of the run was increased.

\section{NUMERICAL RESULTS}

Figure 1 shows the magnetic field diffraction pattern of ZFS1/FS2. In this figure, circles and diamonds are the numerically computed top of the step. Circles were calculated by increasing $\gamma$ at constant $\eta$; diamonds were determined by varying $\eta$ at constant $\gamma$ (as will be seen later, the difference is significant). Small arrows near the diamonds

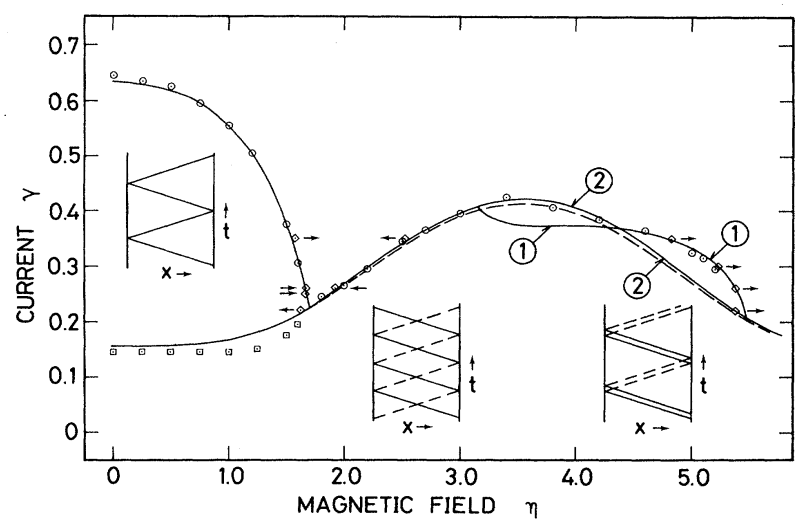

FIG. 1. Magnetic field diffraction pattern of ZFS1/FS2. Circles: step top calculated numerically at constant $\eta$. Squares: step bottom calculated numerically at constant $\eta$. Diamonds: step top calculated numerically at constant $\gamma$. Arrows near diamonds indicate direction of field variation. Solid curve (marked 1 and 2): Enpuku et al. multimode theory. Dashed curve: $\mathrm{Ku}$ lik single-mode theory. Enpuku and Kulik curves coincide at the bottom of the first lobe. Insets show approximate dynamic trajectories in the various $\eta$ regions: solid lines are fluxons or antifluxons; dashed lines are plasma waves.

indicate the direction in which $\eta$ was varied near the maximum point. Beyond the maximum points, the junction switched to a different dynamic state, most often to the McCumber-Stewart hysteresis curve at the corresponding value of $\gamma$. This fact was evidenced by abrupt changes in the value of $\left\langle\phi_{t}\right\rangle$, the voltage waveform, and the corresponding power spectrum. In zero magnetic field, the form of the McCumber-Stewart hysteresis curve can be approximated by ${ }^{15,2}$

$$
\begin{aligned}
& \gamma=4 \alpha E(k) / \pi k, \\
& \left\langle\phi_{t}\right\rangle=\pi / k K(k),
\end{aligned}
$$

where $K(k)$ and $E(k)$ are, respectively, the complete elliptic integrals of the first and second kinds of modulus $k$. From Eqs. (2) it follows that for $k \rightarrow 0, \gamma \rightarrow \alpha\left\langle\phi_{t}\right\rangle$, i.e., the McCumber-Stewart curve approaches asymptotically the Ohmic line, whereas for $k \rightarrow 1,\left\langle\phi_{t}\right\rangle \rightarrow 0$, and $\gamma \rightarrow 4 \alpha / \pi$. Equations (2) continue to hold as a rough approximation even in the presence of magnetic field, at least for $\eta \lesssim 1$.

The squares in Fig. 1 are the numerically computed bottom of the step, calculated by decreasing $\gamma$ at constant $\eta$. As will become clear from Figs. $2-6$, it is possible to determine numerically the precise bottom of the step in the first lobe of the diffraction pattern, but this is no longer the case in the second lobe. Beyond the minimum points, the solution followed the McCumber-Stewart curve down for a certain distance, after which it switched abruptly to a static, zero-voltage state. The lowest $\gamma$ value for which such switching was observed was 0.06 $\leq \gamma<0.07$, which is consistent with the value $4 \alpha / \pi=0.064$ estimated from Eq. (2a).

The dashed curve in Fig. 1 has been calculated from the Kulik theory ${ }^{16}$ for FS2. The input parameter for this 
theory is the quantity $Z_{n}=L^{2} Q_{n} / n^{2} \pi^{2}$, where $n$ is the step number $\left(2\right.$, in our case) and $Q_{n}$ is the quality factor of the $n$th mode. Following Enpuku et al. (Ref. 7) $Q_{n}$ is defined by

$$
\frac{1}{Q_{n}}=\frac{L \alpha}{n \pi}+\frac{n \pi \beta}{L} \text {. }
$$

Insertion of parameter values thus yields $Z_{2}=1.2867$. The Kulik theory gives the maximum height of the step above the Ohmic current. Accordingly, to compare with numerical (or experimental) results it is necessary to add this component to the Kulik value. Since, from our numerical results, the top of FS2 is at $\left\langle\phi_{t}\right\rangle \approx 3.1$, a constant (independent of $\eta$ ) value of $\alpha\left\langle\phi_{t}\right\rangle=0.155$ has been added to the Kulik component in drawing the dashed curve in Fig. 1.

The Kulik theory is seen to predict the maximum points of the second lobe up to $\eta \approx 4$. However, for the maximum points of the first lobe and for the second lobe for $\eta>4$, the theory fails. To predict these results more modes must be included in the computations. Following Enpuku et $a l .{ }^{7}$ we have used five modes with the quality factor $Q_{n}$ given by Eq. (3) with $n=1,2, \ldots, 5$. We have solved Eqs. (10)-(12) in Ref. 7 by means of a standard routine. ${ }^{17}$ The results are shown as the solid curve in Fig. 1. The Enpuku theory is seen to predict the maximum points of the first lobe very well. The maximum values on the second lobe up to $\eta=5.5$ are also in agreement. However, between $\eta=3.1$ and 5.5 the Enpuku theory predicts two curves for the maximum values. The curves are marked 1 and 2 in accordance with a major contribution to the solution from the first and second cavity modes respectively. Curve 2 lies close to the Kulik curve, in agreement with the fact that this latter curve was computed by means of mode 2 exclusively. The numerically computed maximum values (circles) agree with the upper curves (i.e., curve 2 in the interval $\eta=3.1-4.4$ and curve 1 in the interval $\eta=4.4-5.5$ ). Just above the lower curves (i.e., curve 1 for $\eta=3.1-4.4$ and curve 2 for $\eta=4.4-5.5$ ) the computer results exhibit changes in the contents of cavity modes from mode 1 to mode 2 above curve 1 and vice versa above curve 2 . The accompanying hysteresis phenomena are discussed below.

Figures 2-6 depict five vertical (constant- $\eta$ ) slices through the diffraction pattern. Figure 2(a) shows the current dependence of the power levels of the first two Fourier harmonics of the voltage at the left end of the junction in zero magnetic field. Power levels are given by $10 \ln |A|^{2}+100, A$ being the voltage Fourier component. The ac components are thus arbitrarily normalized to $\phi_{t}^{2}=10^{-10}$. Since no loading effects are included in the model, all power levels calculated should be considered ideal, available values. Figure 2(b) shows the current dependence of the first harmonic frequency $f_{1}$ of the oscillation. Since average voltage and frequency are proportional (in steady state) through the relations $\left\langle\phi_{t}\right\rangle=4 \pi f_{1}=2 \pi f_{2}$, Fig. 2 (b) is effectively the currentvoltage characteristic of the step. We have chosen to plot this characteristic in frequency rather than in voltage because in the laboratory, frequency can be measured much more precisely (although perhaps less easily) than voltage.

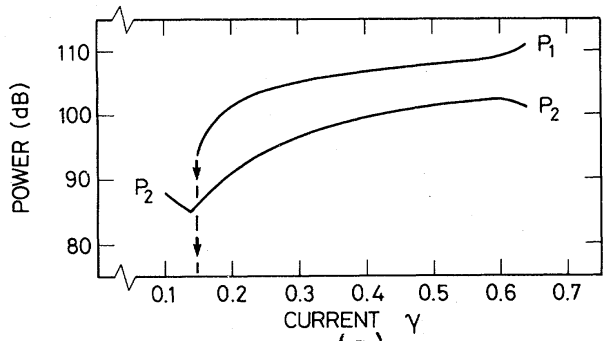

(a)

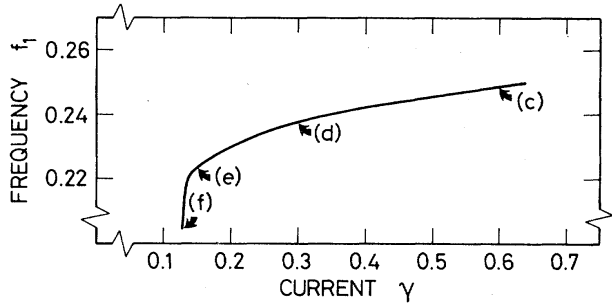

(b)
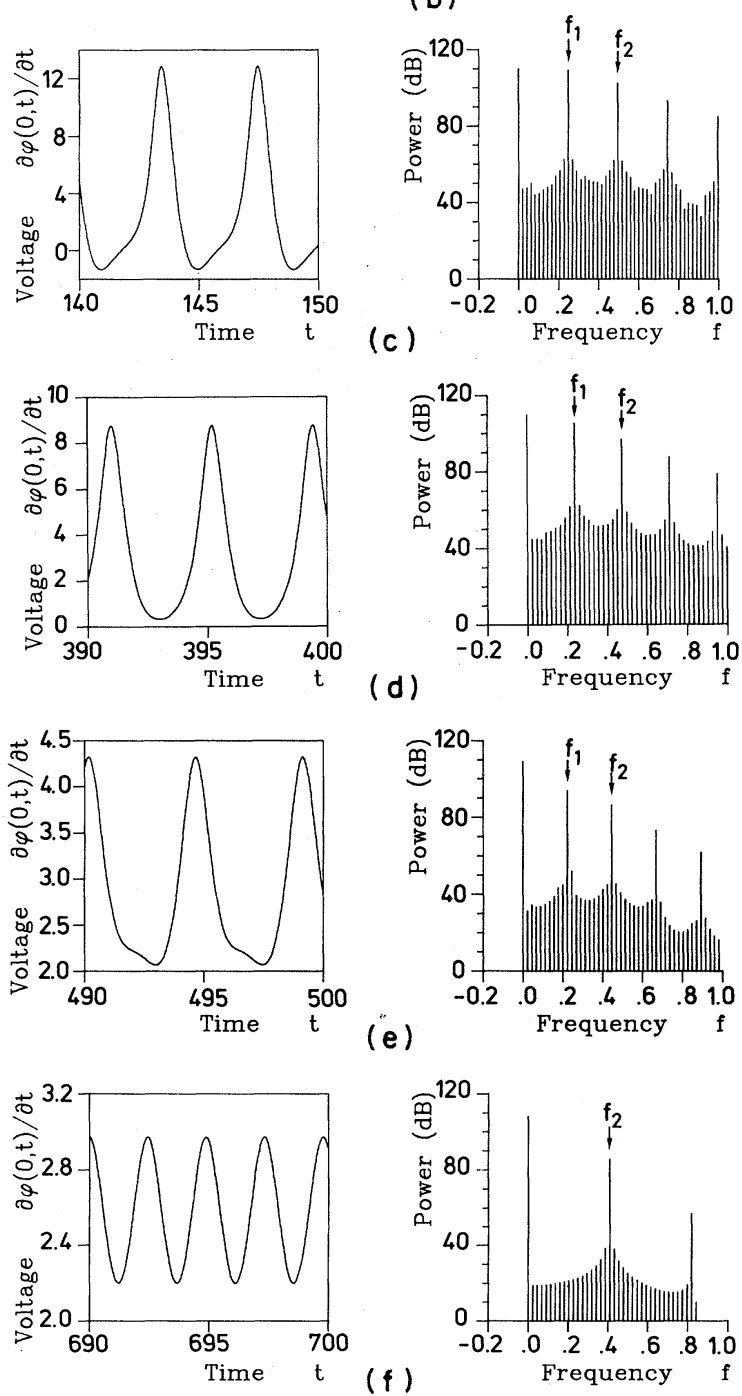

FIG. 2. Section through diffraction pattern at $\eta=0$. (a) Power levels of first two Fourier voltage components at $x=0$, normalized to $\phi_{t}^{2}=1.0 \times 10^{-10}$. (b) First-harmonic frequency. Voltage waveform at $x=0$ and corresponding power spectrum are shown for (c) $\gamma=0.60$, (d) $\gamma=0.30$, (e) $\gamma=0.15$, and (f) $\gamma=0.13$. 


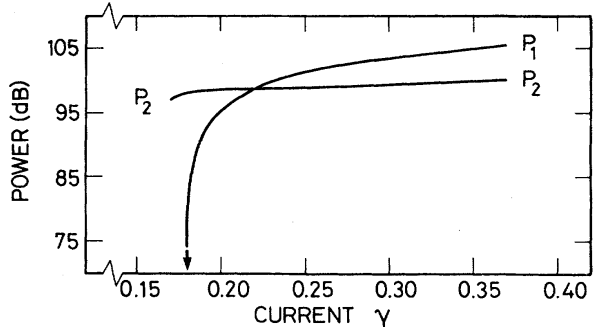

(a)

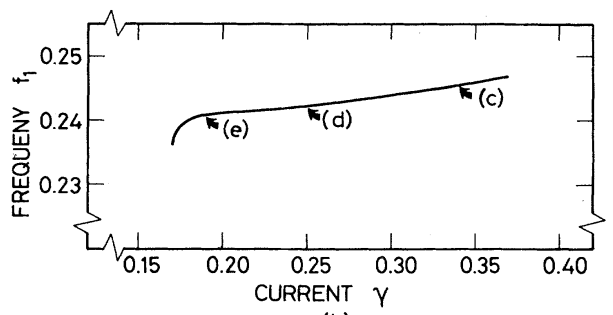

(b)
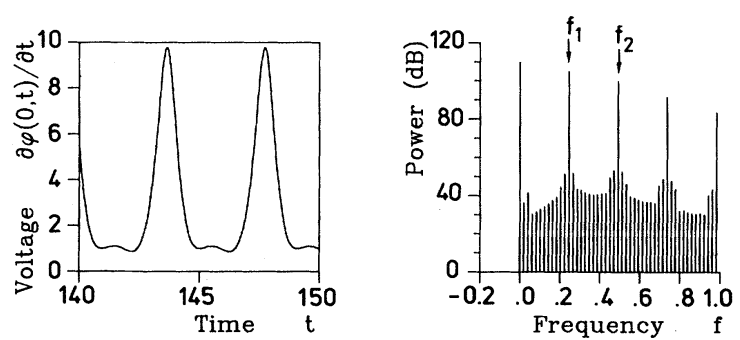

(c)
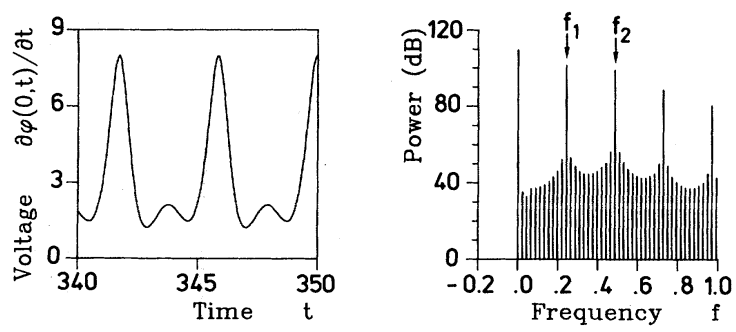

(d)
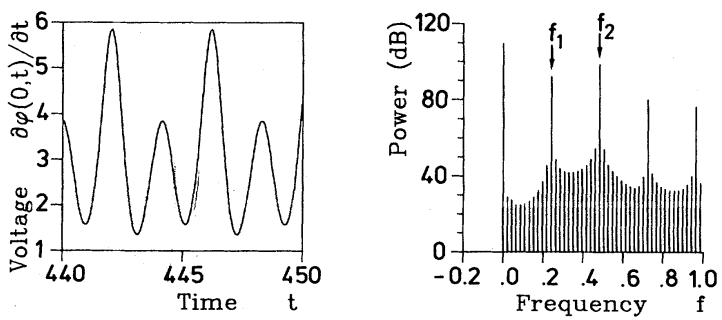

(e)

FIG. 3. Section through diffraction pattern at $\eta=1.5$. (a) Power levels of first two Fourier voltage components at $x=0$, normalized to $\phi_{t}^{2}=1.0 \times 10^{-10}$. (b) First-harmonic frequency. Voltage waveform at $x=0$ and corresponding power spectrum are shown for (c) $\gamma=0.34$, (d) $\gamma=0.25$, and (e) $\gamma=0.19$.
Figures 2(c)-(f) show the voltage waveform and part of the corresponding power spectrum at the four points indicated by arrows in Fig. 2(b). A comparison of Figs. 2(a)-2(e) with results from the literature [see, in particular, Fig. 17 of Ref. 9 and Fig. 5(a) of Ref. 2] leaves no doubt that the oscillation depicted in Fig. 2 is the fluxon oscillation corresponding to ZFS1. Finally, a comparison of Figs. 2(e) and 2(f) makes clear why it is possible to determine precisely the bottom of the step in the first lobe of the diffraction pattern: At a certain value of the bias current $\left(0.14 \leq \gamma<0.15\right.$ in the present case) the $f_{1}$ component of the oscillation abruptly disappears, and a new oscillation evolves for which the dominant component is at $f_{2}$.

Figure 3 shows a similar vertical slice through the diffraction pattern at a point near the right-hand extremity

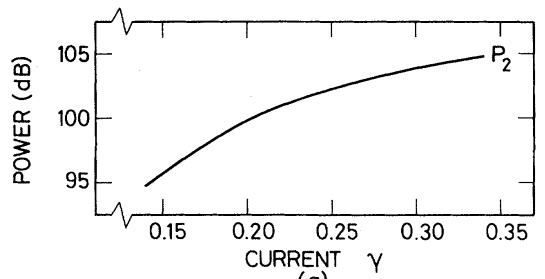

(a)

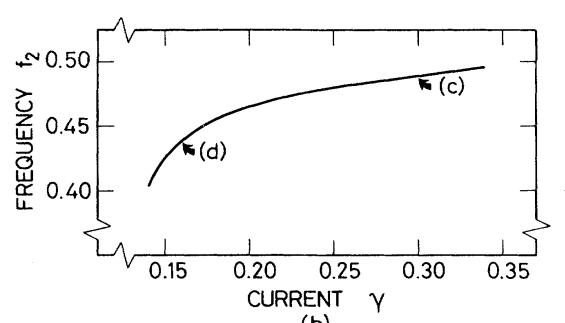

(b)
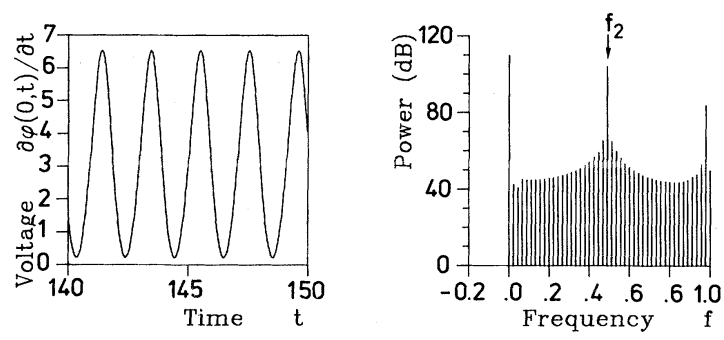

(c)
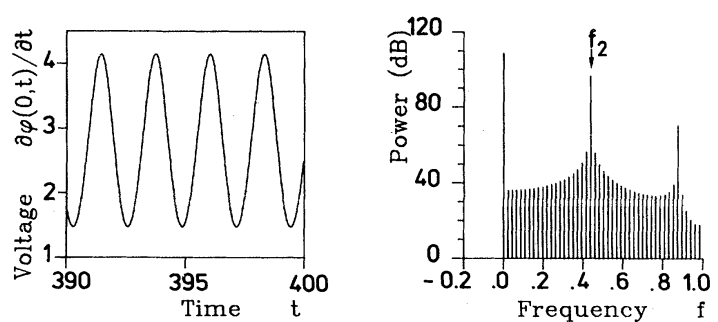

(d)

FIG. 4. Section through diffraction pattern at $\eta=2.5$. (a) Power levels of second Fourier voltage component at $x=0$, normalized to $\phi_{t}^{2}=1.0 \times 10^{-10}$ ( $P_{1}$ is absent). (b) Second-harmonic frequency. Voltage waveform at $x=0$ and corresponding power spectrum are shown for (c) $\gamma=0.30$ and (d) $\gamma=0.16$. 
of the first lobe $(\eta=1.5)$. Here, the behavior of the oscillation near the top of the step is qualitatively quite similar to that shown in Fig. 2. As $\gamma$ is decreased, however, a marked difference is seen: The ratio of the $f_{1}$ power $P_{1}$ to the $f_{2}$ power $P_{2}$ steadily decreases until, near the bottom of the step, $P_{2}$ in fact dominates. Since $f_{2}$ is the dominant frequency of FS2, the nature of the step at $\eta=1.5$ may be described as ZFS1-like near the top and FS2-like near the bottom. It should be noted, however, that the transition between the two oscillation configurations is here relatively gradual, rather than abrupt.

Figure 4 shows a vertical slice at $\eta=2.5$, in the lefthand side of the second lobe of the diffraction pattern. Here the oscillation is purely FS2-like over the entire extent of the step. The power $P_{1}$ is lost in the background

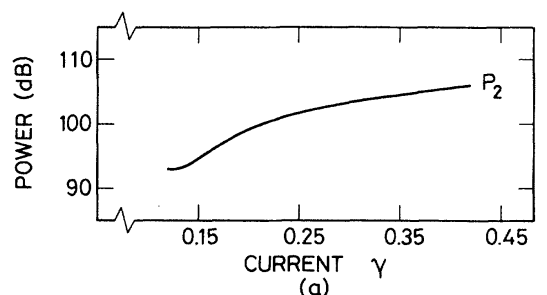

(a)

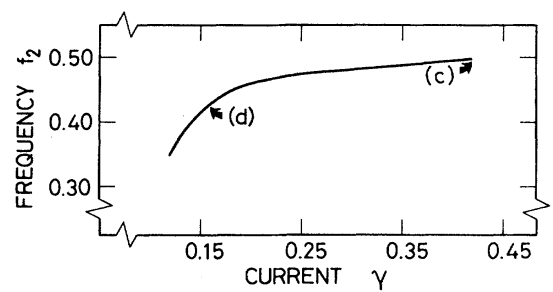

(b)
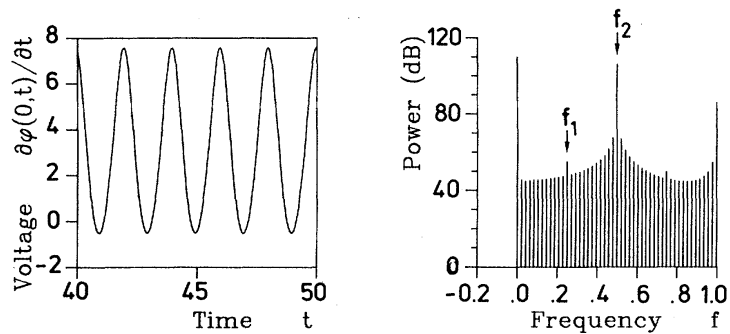

(c)
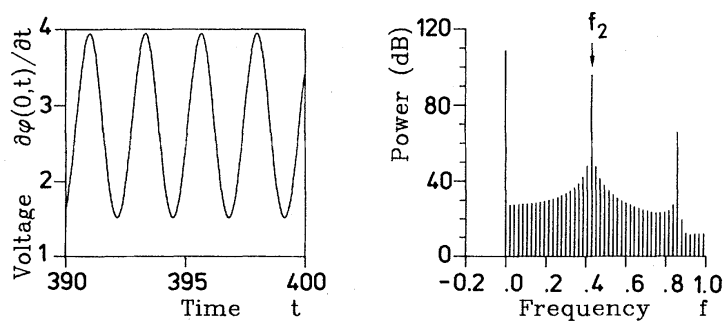

(d)

FIG. 5. Section through diffraction pattern at $\eta=3.4$. (a) Power levels of second Fourier voltage component at $x=0$, normalized to $\phi_{t}^{2}=1.0 \times 10^{-10}$ ( $P_{1}$ is absent or very small). (b) Second-harmonic frequency. Voltage waveform at $x=0$ and corresponding power spectrum are shown for (c) $\gamma=0.42$ and (d) $\gamma=0.16$ noise (the noise here is of numerical, not physical, origin), and $P_{2}$ is the dominant component. The fact that the next-highest component, at $f=2 f_{2}$, lies $20 \mathrm{~dB}$ or more below $P_{2}$, explains why the Kulik theory, which assumes

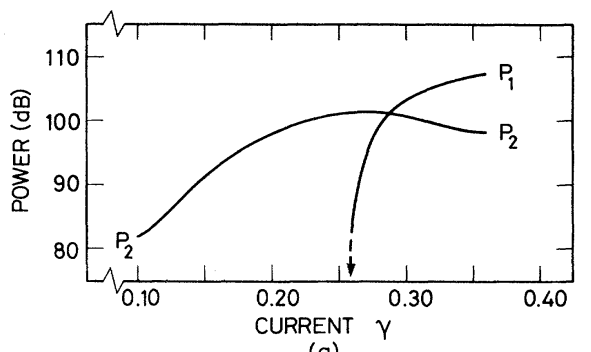

(a)

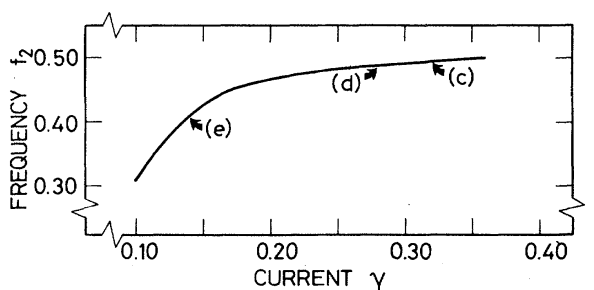

(b)
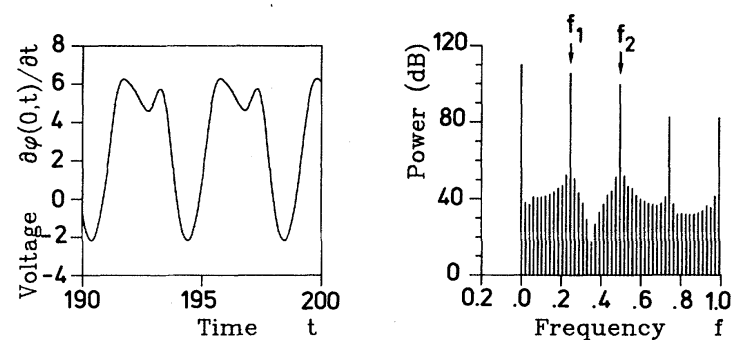

(c)
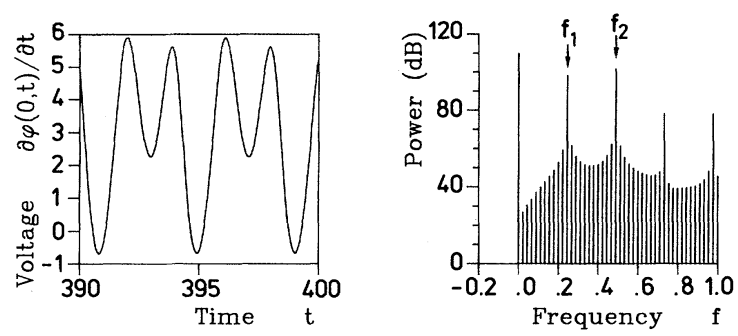

(d)
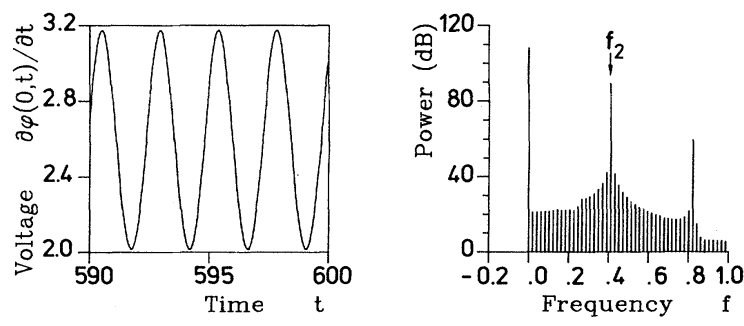

(e)

FIG. 6. Section through diffraction pattern at $\eta=4.6$. (a) Power levels of first two Fourier voltage components at $x=0$, normalized to $\phi_{t}^{2}=1.0 \times 10^{-10}$. (b) Second-harmonic frequency. Voltage waveform at $x=0$ and corresponding power spectrum are shown for (c) $\gamma=0.32$, (d) $\gamma=0.28$, and (e) $\gamma=0.14$. 
a single mode at $f=f_{2}$, gives a reasonable description of the oscillation in this region, as seen in Fig. 1. The difficulty in determining precisely the bottom of the step in the second lobe is now also apparent: $f_{2}$ is the dominant component of both FS2 and the McCumber-Stewart curve. As $\gamma$ is decreased, the step here merges gradually with the McCumber-Stewart curve without any abrupt changes, in contrast with what happens in the first lobe.

The behavior of the oscillation along a vertical slice at the peak of the second lobe $(\eta=3.4)$ is shown in Fig. 5. Here, the situation is very similar to that depicted in Fig. 4 except at the very top of the step, where, with increasing $\gamma$, the $f_{1}$ component just begins to emerge from the background, as seen in Fig. 5(c).

This tendency continues more markedly in Fig. 6, which shows a vertical slice at $\eta=4.6$. Here, recalling the situation depicted in Fig. 3, the step is ZFS1-like near the top and FS2-like near the bottom. As in Fig. 3, the transition here between the two oscillation configurations is relatively smooth.

The information contained in Figs. $2-6$ is summarized in a highly schematic and approximate fashion in the insets of Fig. 1. These show trajectories in the $x-t$ plane, with fluxons and antifluxons indicated by solid lines and localized plasma waves indicated by dashed lines. The locations in $\eta$ of the three insets in Fig. 1 indicate roughly the regions where the corresponding dynamic configurations are observed (we emphasize once again, however, that the transitions between the various configurations are gradual).

A rather different perspective on the nature of the oscillations is obtained by taking horizontal (constant- $\gamma$ ) slices through the diffraction pattern. Two such slices, at $\gamma=0.35$ and 0.26 , are shown in Figs. 7 and 8. Figure 7 shows the magnetic field dependence of the frequency $f_{2}$ (proportional to the voltage) in the two slices, while Fig. 8 shows the corresponding behavior of the power levels, $P_{1}$ and $P_{2}$, at $f_{1}$ and $f_{2}$. The salient facts that emerge from these two figures may be summarized as follows: (i) The essential, overall dependence of the oscillation frequency on magnetic field is the inverse (qualitatively) of that of the diffraction pattern; where the height of the step decreases with field, the frequency increases, and vice versa.

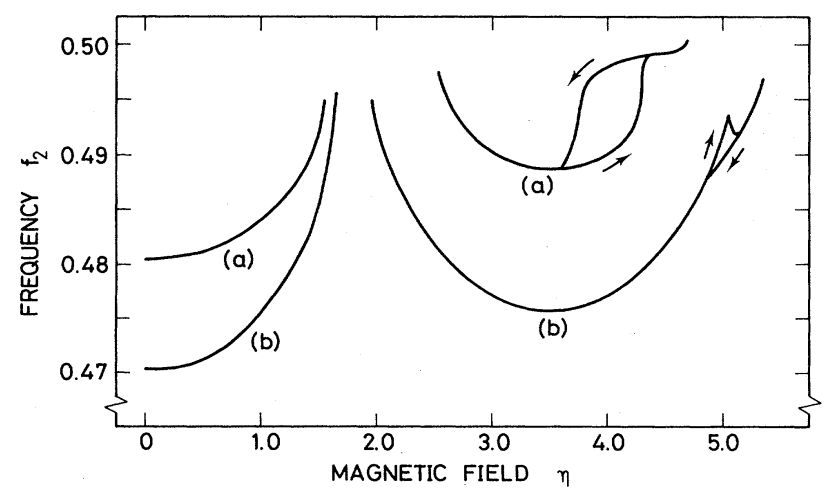

FIG. 7. Second-harmonic frequency section through diffraction pattern at (a) $\gamma=0.35$ and (b) $\gamma=0.26$. Arrows indicate hysteresis.

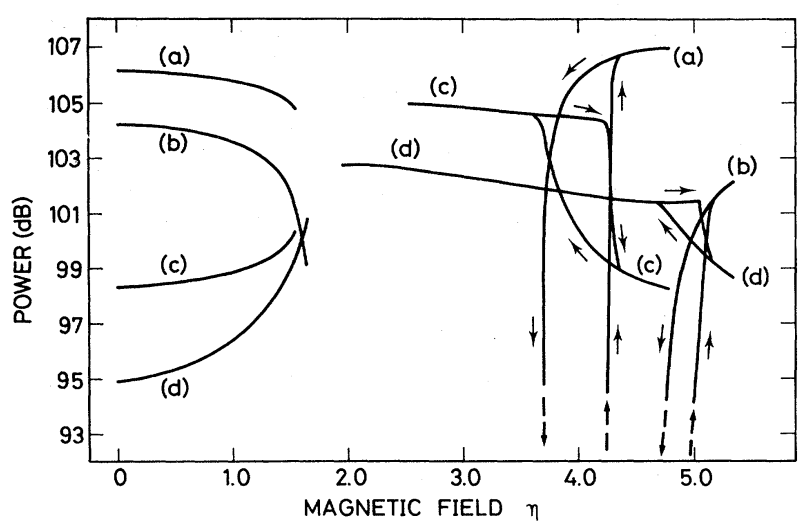

FIG. 8. Power section through diffraction pattern at constant $\gamma$. Powers measured at $x=0$ and normalized to $\phi_{t}^{2}$ $=1.0 \times 10^{-10}$. (a) First harmonic $P_{1}$ at $\gamma=0.35$. (b) First harmonic $P_{1}$ at $\gamma=0.26$. (c) Second harmonic $P_{2}$ at $\gamma=0.35$. (d) Second harmonic $P_{2}$ at $\gamma=0.26$. Arrows indicate hysteresis.

(ii) In the first lobe of the diffraction pattern the behavior is quite regular; the frequency increases monotonically with field, and $P_{1}$ is the dominant component. We note parenthetically here that this frequency behavior may be different for longer junctions. ${ }^{18,19}$ (iii) In the left half of the second lobe the behavior is also regular; the frequency

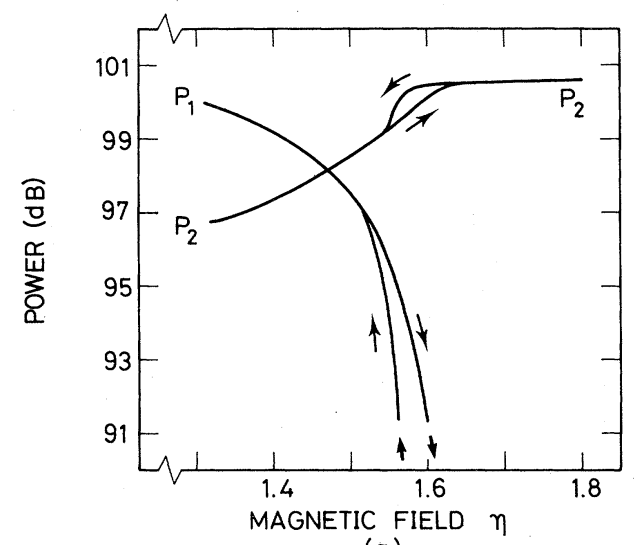

(a)

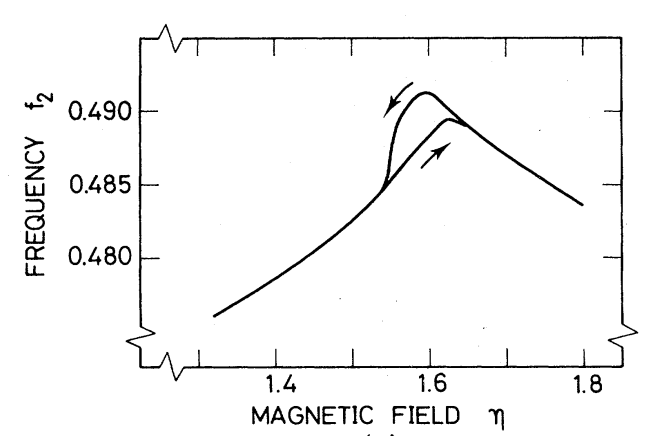

(b)

FIG. 9. Partial section through diffraction pattern at $\gamma=0.21$. (a) Power levels of first two Fourier voltage components at $x=0$, normalized to $\phi_{t}^{2}=1.0 \times 10^{-10}$. (b) Secondharmonic frequency. Arrows indicate hysteresis. 
here decreases monotonically with field, reaching a minimum in correspondence with the peak of the second lobe, and $P_{2}$ is strongly dominant (the $f_{1}$ component lies within the background noise). (iv) In the right half of the second lobe $P_{1}$ once again becomes dominant. The crossover point, where $P_{1} \approx P_{2}$, occurs at larger values of $\eta$ for decreasing $\gamma$. These observations suggest that it might in fact be more appropriate to refer here to a third lobe, rather than to a half of the second lobe. (v) A strongly hysteretic behavior of both frequency and power is observed in the right half of the second lobe (i.e., third lobe). This fact may explain why experimental measurements [see, e.g., Paternò and Nordman ${ }^{20}$ as well as Fig. 12(a) below] often display notable irregularities in this region.

Figure 9 shows a small section of a horizontal slice at $\gamma=0.21$ in the region just under the juncture point of the first and second lobes. Since $\gamma=0.26$ and 0.35 both lie above this juncture point, the curves in Figs. 7 and 8 are discontinuous in this region. The major conclusions to be drawn from Fig. 9 are similar to those drawn above: (i) The qualitative shape of the field dependence of the oscillation frequency is the inverse of that of the diffraction pattern. (ii) Hysteresis is observed in $\eta$ regions where the dominant mode is changing.

\section{EXPERIMENTAL RESULTS}

The numerical calculations discussed above were compared with measurements on overlap geometry $\mathrm{Nb}-\mathrm{Nb}$ oxide- $\mathrm{Pb}$ junctions having parameters comparable with those used in the calculations. Although the discussion below is appropriate for the many junctions investigated, ${ }^{21}$ two junctions were measured in detail. Junction no. S105-1 has dimensions $479 \times 179 \mu \mathrm{m}^{2}$, maximum zero-voltage current $I_{c 0}=1.40 \mathrm{~mA}$, and an estimated normalized length, $L$, of 2 . Junction no. $65 \mathrm{H} 7$ has dimensions $467 \times 67 \mu \mathrm{m}^{2}, I_{c 0}=0.53 \mathrm{~mA}$, and $L$ slightly less than 2 . From independent measurements on similar junctions an estimate of $\alpha$ and $\beta$ can be made. ${ }^{22}$ The estimate is reasonably consistent with the values $\alpha=0.05$ and $\beta=0.02$ used in the calculations, although the experimental values are probably somewhat smaller. The parameter values of both junctions are such that the fundamental soliton frequency $f_{1}$ may be detected with an $X$-band receiver $(8-12 \mathrm{GHz})$. Any radiation at $f_{2}$, however, is outside the frequency band of the detector used. The microwave receiver had an overall noise figure of about 8 dB. By using a spectrum analyzer both the power and the frequency of microwave signals from the junction could be measured. Generally, the received power was $25 \mathrm{~dB}$ or less above the physical noise level of the receiver. All data discussed here were taken at $4.2 \mathrm{~K}$.

To investigate the fluxon dynamics the following measurements were performed: (i) $I-V$ curves of the steps. (ii) The magnetic field dependence of the maximum height of the steps. (iii) The magnetic field dependence of the voltage of the steps with the bias current as a parameter. (iv) The power and frequency of the $f_{1}$ radiation emitted from the junction.

Figure 10 shows the low-voltage part of the $I-V$ curve in zero magnetic field of junction no. S10-5-1. Three

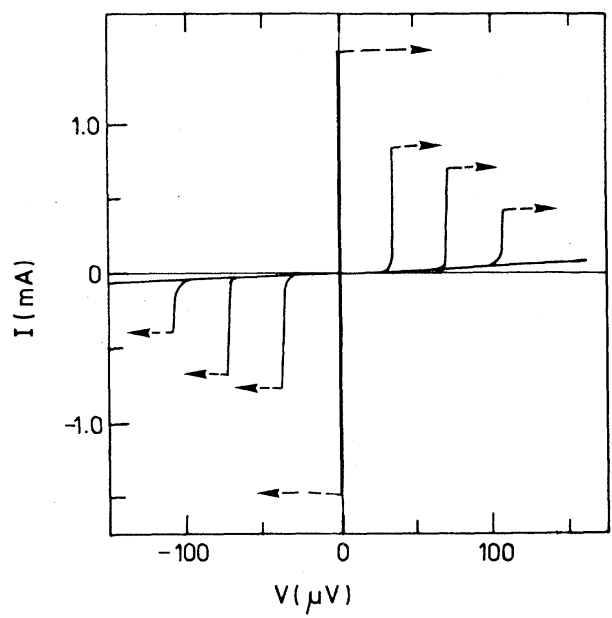

FIG. 10. $I-V$ curve of junction no. S10-5-1, showing three zero-field steps. The dashed curves indicate switching.

ZFS's are seen. The high-voltage part omitted in Fig. 10 only shows the usual increase in current at the energy gap voltage. The dashed lines show switching at the top of the supercurrent and the three ZFS's. The $I-V$ curve of the ZFS's is obtained by decreasing the current to obtain a bias point just below the foot of the ZFS's and then increasing the current again. This $I-V$ curve is typical of the samples investigated. Although not shown with sufficient voltage resolution, the shape of ZFS1 may be compared with Fig. 2(b).

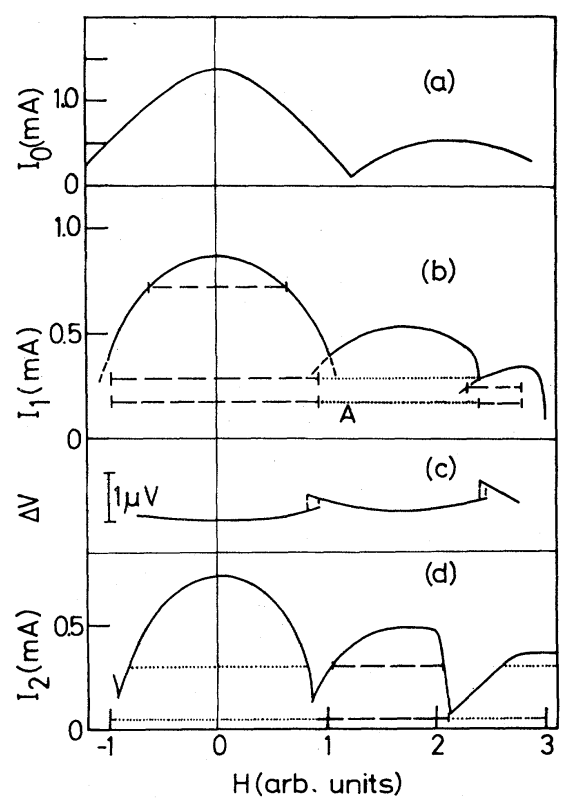

FIG. 11. Junction no. S10-5-1. (a) Magnetic field dependence of the maximum zero-voltage current. (b) Magnetic field dependence of the height of ZFS1/FS2. (c) Voltage tuning of ZFS1/FS2 corresponding to bias level $A$ in (b). (d) Magnetic field dependence of the height of ZFS2/FS4. The dashed lines in (b) and (d) show examples of parameter regions where $f=f_{1}$ radiation was observed. The dotted lines show parameter regions where $f=f_{1}$ radiation was not observed. 
Figures 11(a) and 11(b) show the magnetic field dependence of the maximum zero-voltage current and of ZFS1/FS2, respectively. Note that the lobe pattern of ZFS1/FS2 is in good qualitative agreement with the calculated one in Fig. 1. In fact, the agreement here is at least semiquantitative: The ratio of the zero-field current in Fig. 11(b) to that in Fig. 11(a) is 0.63 ; the corresponding value from Fig. 1 is $0.64<\gamma \leq 0.65$. The extrapolated zero of the first lobe in Fig. 11(b) occurs at a field value approximately equal to that seen for the extrapolated zero of the first lobe in Fig. 11(a) (this is a generally observed experimental fact). In terms of the normalized field $\eta$, the extrapolated zero of the first lobe of the zero-voltage current occurs at $\eta=2$ for very long junctions (the exact value for shorter junctions, which is the same for the overlap and the in-line geometries, may be calculated from the theory of Owen and Scalapino ${ }^{23}$ ). Extrapolating to $\gamma=0$, the first lobe in Fig. 1 also yields $\eta \approx 2$. The dashed lines show examples of parameter regions where $f=f_{1}$ radiation (at approximately $9 \mathrm{GHz}$ ) was observed. In general, the $f=f_{1}$ radiation was observed in the first and third lobes but not in the second lobe. This is in agreement with the results of the numerical calculations, in particular Fig. 8. By comparison with the calculations the reason for the absence of radiation in the second lobe (FS2) is that here the $f=f_{2}$ radiation is at presumably $\sim 18 \mathrm{GHz}$, outside the range of the receiver. Figure 11(c) shows, for a bias current corresponding to $A$ in Fig. 11(b), the magnetic field tuning of the voltage of ZFS1/FS2 (the absolute value of the voltage is approximately $35 \mu \mathrm{V}$ ). Figure 11(c) is in good qualitative agreement with the calculation of Fig. 7, showing both the increase in frequency (voltage) as the border regions of the lobes are approached, and the hysteresis there. From the voltage curve, however, it cannot be decided whether it is the $f=f_{1}$ or the $f=f_{2}$ radiation that is dominant. Thus, determination of the fluxon-mode configuration requires a measurement of the frequency of the emitted microwave radiation. Such a measurement is described below (Fig. 12). Figure 11(d) shows the lobe pattern of the second step although no corresponding numerical calculations have been performed for this step. The dashed lines show examples of parameters where $f=f_{1}$ radiation was observed. In general, no such radiation was observed in the first and third lobes; however, $f=f_{1}$ radiation was observed everywhere in the second lobe. Most likely, the soliton configuration in the first lobe is that of a symmetric fluxon-antifluxon mode (with $f=f_{2}$ ). This is in apparent contradiction with measurements on other samples $^{4}$ where the $f=f_{1}$ radiation was also measured on ZFS2 in zero magnetic field, but the difference may simply be a manifestation of the two soliton configurations, symmetric $\left(f=f_{2}\right)$ and bunched $\left(f=f_{1}\right)$, that have been demonstrated numerically for ZFS2.9 On junction no. $65 \mathrm{H7}$, in fact, the bunched mode was observed on ZFS2. In the second lobe (FS4) a three-fluxon-one-antifluxon mode would give the right frequency $\left(f_{1}\right)$ and voltage; however, other configurations are also possible.

Figure 12(a) shows the diffraction pattern for ZFS1/FS2 of junction no. 65H7. Qualitatively, it is quite similar to Fig. 11(b); however, the right half of the second

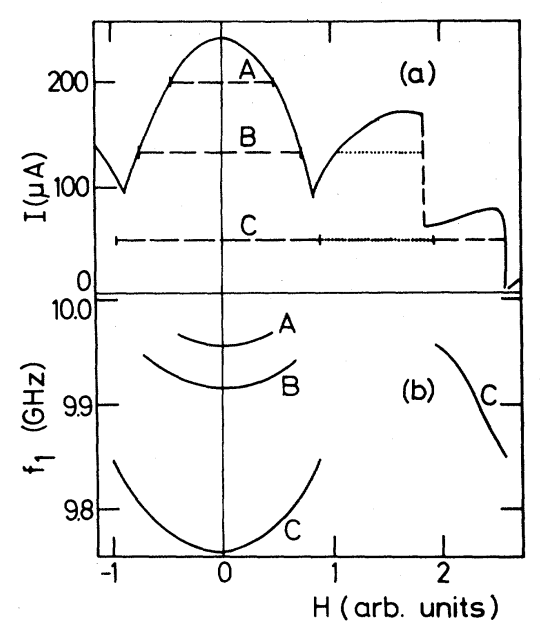

FIG. 12. Junction no. 65H7. (a) Magnetic field dependence of the height of ZFS1/FS2. The dashed lines show examples of parameters where $f=f_{1}$ radiation was observed. The dotted lines show parameter regions where $f=f_{1}$ variation was not observed. (b) Frequency of the $f=f_{1}$ radiation corresponding to bias levels $A, B, C$ in (a).

lobe appears somewhat anomalous. For this measurement the hysteresis phenomena between lobes were observed but not carefully mapped. As indicated by the lines $A, B, C$ for different constant bias currents, $f=f_{1}$ radiation was observed in the first and third lobes, but not in the second. Figure 12(b) shows a measurement of the frequency of the emitted $f=f_{1}$ radiation corresponding to bias currents $A, B, C$ in Fig. 12(a). The positive frequency tuning in the first lobe is in good agreement with Fig. 7. The absence of the $f=f_{1}$ radiation in the second lobe and the reappearance of such radiation at the transition between the second and third lobes are also in qualitative agreement with the numerical calculation of Fig. 8. As noted in connection with Figs. 7 and 9 the frequency tuning resembles the inverse of the diffraction pattern. Qualitatively, curve $C$ in Fig. 12(b) is also in agreement with the voltage tuning in the first and third lobes of Fig. 11(c).

\section{CONCLUSIONS}

The intermediate regime $L \gtrsim 1$, where the standard analytical methods (cavity-mode theory for $L \lesssim 1$ and soliton perturbation theory for $L \gg 1$ ) a priori do not apply, was investigated numerically and experimentally. A comparison between the experiments and the numerical calculations showed a very good qualitative agreement. Based on this comparison it was possible to identify the various soliton modes in the magnetic field lobes of ZFS1/FS2. The extension of single-cavity-mode theory to multimodes due to Enpuku et al. ${ }^{7}$ gave satisfactory predictions of the diffraction pattern for ZFS1 and FS2. Thus, with caution elements from both types of theories are usable, however, numerical simulation is necessary for a wider understanding of experimental observations. 


\section{ACKNOWLEDGMENTS}

It is our pleasure to thank Alwyn C. Scott for many helpful discussions. The financial support of the Danish
Natural Science Research Council, the Thomas B. Thriges Fund, and the European Research Office of the United States Army (through Contract No. DAJA-37-82-C-0057) is gratefully acknowledged.
${ }^{1}$ N. F. Pedersen, in Advances in Superconductivity, NATO Advanced Study Institute Series, B100, edited by B. Deaver and John Ruvalds (Plenum, New York, 1982), p. 149.

${ }^{2}$ S. N. Erné and R. D. Parmentier, J. Appl. Phys. 51, 5025, (1980).

${ }^{3}$ S. N. Erné, A. Ferrigno, and R. D. Parmentier, Phys. Rev. B 27, 5440 (1983).

${ }^{4}$ B. Dueholm, O. A. Levring, J. Mygind, N. F. Pedersen, O. H. Soerensen, and M. Cirillo, Phys. Rev. Lett. 46, 1299 (1981).

${ }^{5}$ E. Joergensen, V. P. Koshelets, R. Monaco, J. Mygind, M. R. Samuelsen, and M. Salerno, Phys. Rev. Lett. 49, 1093 (1982).

${ }^{6}$ D. W. McLaughlin and A. C. Scott, Phys. Rev. A 18, 1672 (1978).

${ }^{7}$ K. Enpuku, K. Yoshida, and F. Irie, J. Appl. Phys. 52, 344 (1981).

${ }^{8}$ M. Cirillo, R. D. Parmentier, and B. Savo, Physica (Utrecht) 3D, 565 (1981).

${ }^{9}$ P. S. Lomdahl, O. H. Soerensen, and P. L. Christiansen, Phys. Rev. B 25, 5737 (1982).

${ }^{10}$ A. Matsuda and T. Kawakami, Phys. Rev. Lett. 51, 694 (1983).

${ }^{11}$ S. Sakai, H. Akoh, and H. Hayakawa, Jpn. J. Appl. Phys. 22,
L479 (1983).

${ }^{12}$ A. Barone, W. J. Johnson, and R. Vaglio, J. Appl. Phys. 46, 3628 (1975).

${ }^{13}$ M. P. Soerensen, P. L. Christiansen, R. D. Parmentier, and O. Skovgaard, Appl. Phys. Lett. 42, 739 (1983).

${ }^{14}$ IMSL, Inc. (Houston, Texas) International Mathematical and Statistical Library (Ed. 9) routine FFTRC, 1982 (unpublished).

${ }^{15}$ W. A. Schlup, J. Phys. C 7, 736 (1974).

${ }^{16}$ I. O. Kulik. Zh. Tekn. Fiz. 37, 157 (1967) [Sov. Phys.-Tech. Phys. 12, 111 (1967)].

${ }^{17}$ IMSL, Inc. (Houston, Texas) International Mathematical and Statistical Library (Ed. 9) routine ZSCNT, 1982 (unpublished).

${ }^{18}$ S. N. Erné, A. Ferrigno, S. DiGenova, and R. D. Parmentier, Lett. Nuovo Cimento 34, 121 (1982).

${ }^{19}$ O. A. Levring, N. F. Pedersen, and M. R. Samuelsen, J. Appl. Phys. 54, 987 (1983).

${ }^{20}$ G. Paternò and J. Nordman, J. Appl. Phys. 49, 2456 (1978).

21 Junctions fabricated both at the Technical University of Denmark and at the University of Salerno were measured, with similar results.

${ }^{22}$ N. F. Pedersen and D. Welner, Phys. Rev. B 29, 2551 (1984).

${ }^{23}$ C. S. Owen and D. J. Scalapino, Phys. Rev. 164, 538 (1967). 